\title{
Numerical Analysis of Metal-Cylinder-Based Microwave Plasma Module
}

\author{
M. SoBAŃSKI ${ }^{a}$, M. JASIŃSKI ${ }^{a, *}$, AND J. MIZERACZYK ${ }^{a, b}$ \\ ${ }^{a}$ Centre for Plasma and Laser Engineering, The Szewalski Institute of Fluid-Flow Machinery \\ Polish Academy of Sciences, J. Fiszera 14, 80-231 Gdańsk, Poland \\ ${ }^{b}$ Dept. of Marine Electronics, Gdynia Maritime University, Morska 81-87, 81-255 Gdynia, Poland
}

\begin{abstract}
We present optimization of energy transfer in the waveguide-supplied metal-cylinder-based microwave plasma module with an inner cylindrical quartz tube. The construction of microwave plasma module is based on a WR 340 waveguide standard. Presented microwave plasma module operates at atmospheric pressure and frequency of $2.45 \mathrm{GHz}$. There is a reduced height section waveguide in microwave plasma module which provides local increase of the electric field in the plasma region. Microwave plasma module is terminated with a movable plunger which plays the role of the tuning element. Tuning characteristics of microwave plasma module are defined as the dependence of the $P_{R} / P_{I}$ on the position $l_{s}$ of the movable plunger, where $P_{R}$ and $P_{I}$ are the microwave power reflected and power incident, respectively. The powers $P_{R}$ and $P_{I}$ are measured in the input plane of microwave plasma module. The purpose of the presented optimization is to achieve $P_{R}$ lower than $5 \%$ of $P_{I}$ in widest range of position of movable plunger $l_{s}$. We used Comsol Multiphysics software to make numerical analysis which allows to optimize the parameters of microwave plasma module. Results of numerical analysis show that $P_{R} / P_{I}$ is lower than 0.05 in a wide range of movable plunger position $l_{s}$.
\end{abstract}

DOI: 10.12693/APhysPolA.125.1309

PACS: 88.80.hp, 84.40.-x, 52.35.Hr, 84.40.Dc, 02.70.Dh, 88.30.E-, 52.65.-y, 52.50.Dg, 11.55.-m, 52.40.Fd, 84.40.Az

\section{Introduction}

Microwave plasma sources (MPSs) are used for gas and water processing [1-3], synthesis of carbon nanotubes [4]. The MPS presented in this paper is an improved version of the existing MPS used for hydrogen production via conversion of hydrocarbons [5,6]. This MPS called microwave plasma module (MPM) operates at atmospheric pressure and frequency of $2.45 \mathrm{GHz}$.

About $70 \%$ of an electric energy delivered from power network can be converted into microwave energy in MPM. This paper presents optimization of this $70 \%$ of energy which depends on parameters described in next section. Efficiency of hydrogen production increases if the absorbed microwave power $P_{A}$ in the plasma increases $\left(P_{A} \approx P_{I}-P_{R}\right)$. So, it is very important to ensure possible the lowest value of the reflected power $P_{R}$. The purpose of the optimization of the presented MPM is to achieve $P_{R}$ lower than $5 \%$ of $P_{I}$ in widest range of movable plunger position $l_{s}$. Movable plunger plays the role of tuning element. The dependence of $P_{R} / P_{I}$ on the movable plunger position $l_{s}$ is called the tuning characteristic. The tuning characteristic plays role of the energy efficiency indicator of MPM. To calculate optimal values of main parameters of MPM we used Comsol Multiphysics software, which uses finite element method (FEM).

*corresponding author; e-mail: mj@imp.gda.pl

\section{Construction of MPM}

Sketch of the presented MPM is shown in Fig. 1. The presented MPM is based on the standard WR 340 waveguide with an inner width $a=86.4 \mathrm{~mm}$ and height $b=43.2 \mathrm{~mm}$, respectively. The wavelength in this waveguide $\lambda_{\mathrm{g}}$ for basic $H_{10}$ mode is equal to $173.4 \mathrm{~mm}$. MPM operates at atmospheric pressure and frequency of $2.45 \mathrm{GHz}$. MPM is terminated with a movable plunger. Between the plane 1 and plane 4 there is a metal ridge of a width of $w_{1}$ and height of $h_{1}$ inserted into the waveguide. This ridge forms a reduced height section waveguide which provides local increase of the electric field in the plasma region. If the width of the ridge $w_{1}=a$ the wavelength $\lambda_{\mathrm{g} 1}$ in the reduced height section equals $\lambda_{\mathrm{g}}$. If the width of the ridge $w_{1}<a$ the wavelength $\lambda_{\mathrm{g} 1}<\lambda_{\mathrm{g}}$. Ridge waveguides are used to extend working frequency band of microwave devices [7]. Ridge waveguides were used in MPSs to extend range of movable plunger position for which the $P_{R} / P_{I}$ is lower than demanded level [8]. The length of the reduced section of waveguide is equal to $\lambda_{\mathrm{g} 1} / 2$.

In discharge axis (plane 3 ) there are two metal cylinders (upper one and lower one) of the diameter of $47 \mathrm{~mm}$ connected with MPM through the circular gaps in the ridge and waveguide walls. There is a quartz discharge tube mounted in the discharge axis, which prevents the working gas from flowing into the waveguide. The outer diameter and thickness of the quartz tube are $26 \mathrm{~mm}$ and $2 \mathrm{~mm}$, respectively.

In the plane 1 an optional inductive iris with width of $d$ can be inserted. 


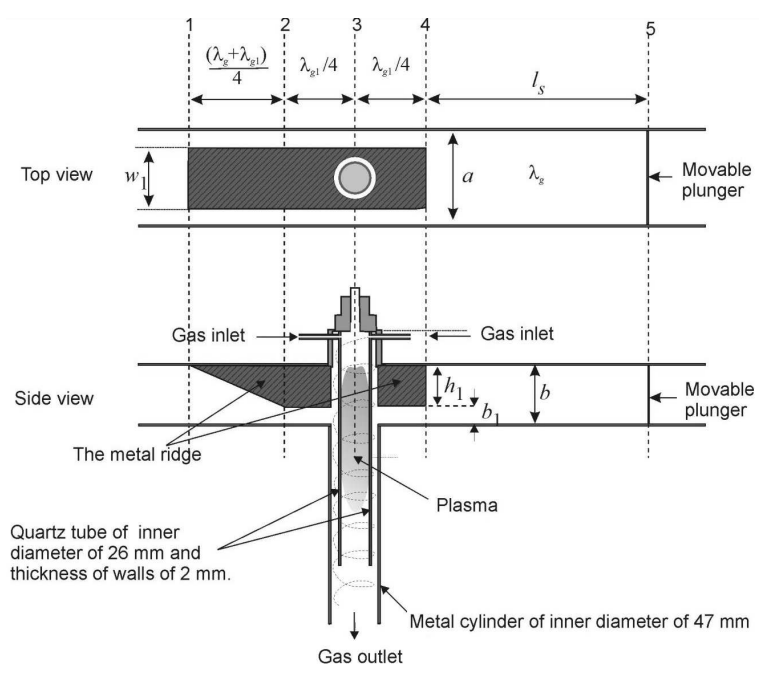

Fig. 1. Sketch of the microwave plasma module (MPM).

Between the plane 1 and plane 2 the metal ridge forms a tapered section which assures smooth transition from the standard waveguide dimensions to the waveguide of reduced height. The length of the tapered section is equal to $\left(\lambda_{\mathrm{g}}+\lambda_{\mathrm{g} 1}\right) / 4$.

A working gas is introduced to MPM by 4 gas inlets mounted tangentially to the upper metal cylinder (Fig. 1). This assures the swirl flow of the working gas, which stabilizes the plasma in the quartz discharge tube.

\section{Assumption data}

The purpose of the numerical analysis is to calculate values of MPM parameters which can assure $P_{R} / P_{I}$ below 0.05 in widest range of normalized movable plunger position. The parameters which were optimized are: the height of the reduced height waveguide $b_{1}=b-h_{1}$, width of the ridge $w_{1}$, and the width $d$ of the iris.

We used a standard model of the plasma as in $[9,10]$, where plasma region is a cylinder filled with uniform medium of the complex electric permittivity given by the Lorentz formula

$$
\varepsilon_{p}=1-n /(1-j s)
$$

where $n=n_{\mathrm{e}} / n_{\mathrm{c}}$ and $s=v / \omega$ are the normalized electron density and collision frequency, respectively, $n_{\mathrm{c}}=$ $\omega^{2} \varepsilon_{0} m / e$ is the critical electron density, $n_{\mathrm{e}}$ is the electron density, $v$ is the electron-neutral collision frequency for momentum transfer, $\omega=2 \pi f$ is the angular frequency.

We assumed that $s=0.1$ and $n$ is in the range 10 to 20 . For these plasma parameters presented above $(s$ and $n$ ) the calculated tuning characteristics of MPM were in good agreement with experimental results [10]. Basing on our experimental observations we assumed that plasma cylinder height and plasma radius are equal to $64 \mathrm{~mm}$ and $10 \mathrm{~mm}$, respectively. The plasma cylinder is localized just under the upper wall of the ridge and penetrates the waveguide and metal cylinder through circular gaps on discharge axis towards the working gas outlet. It is assumed that all metal boundaries are perfect electric conductors. Microwave power of $4 \mathrm{~kW}$ is delivered to the input plane 1 by WR 340 standard waveguide. It was assumed that the electric permittivity of the quartz tube $\varepsilon_{r}=3.8$.

Comsol Multiphysics software allows to calculate the input reflection coefficient of MPM. The value of the $P_{R} / P_{I}$ coefficient for movable plunger position $l_{s}$ can be calculated using formula [11]:

$$
\frac{P_{R}}{P_{I}}=f\left(\frac{l_{s}}{\lambda_{\mathrm{g}}}\right)=\left|\Gamma_{\mathrm{in}}\right|^{2} .
$$

The calculation of $P_{R} / P_{I}\left(l_{s}\right)$ for every single movable plunger position $l_{s}$ is very time-consuming work. We used less time-consuming method proposed in [12], where MPS was treated as two port network terminated with a short circuited transmission line of the length $l_{s}$. The two-port can be represented by its scattering matrix, which can be calculated by Comsol Multiphysics software.

\section{Results of numerical analysis}

In this section we present tuning characteristics of MPM. Figure 2 presents a comparison of the calculated tuning characteristics of MPM before and after optimization. The calculated characteristics for normalized electron density $n$ assumed in the range of $16-16.5$ are in good agreement with the experimental result without ridge and without iris. In the experiment, the gas mixture of $\mathrm{CH}_{4}$ and $\mathrm{CO}_{2}$ was introduced via inlets shown in Fig. 1. The flow rate of each working gas was $50 \mathrm{l} / \mathrm{min}$, and the input microwave power was $4 \mathrm{~kW}$.

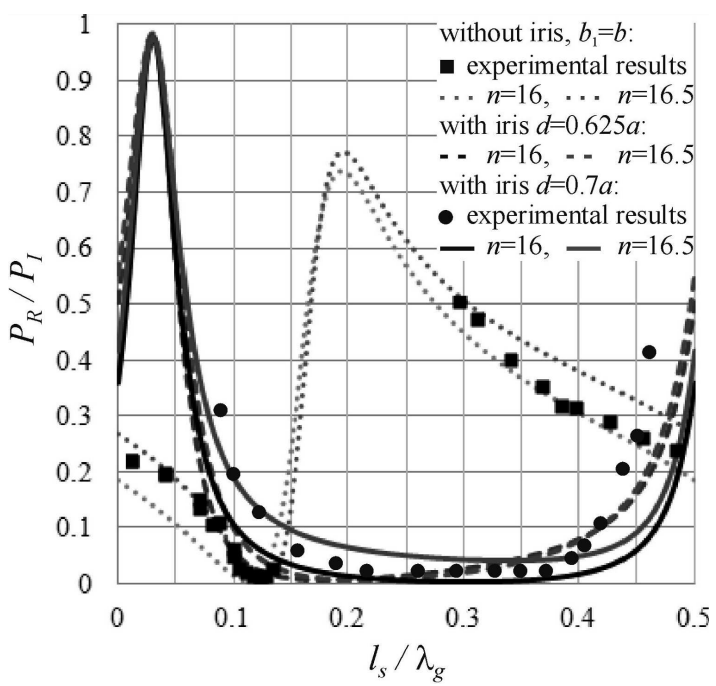

Fig. 2. Tuning characteristics of MPM, before $\left(b_{1}=b\right.$, without iris) and after optimization $\left(b_{1}=0.3 b, w_{1}=\right.$ $0.8 a$, with iris of width $d=0.625 a$ and $d=0.7 a$ ).

Figure 3 presents the tuning characteristics of MPM as the function of two variables: the normalized movable plunger position $\left(l_{s} / \lambda_{\mathrm{g}}\right)$ and normalized electron density $n$.

The tuning characteristic can have values in range of 0 to 1, but in Fig. 3 and Fig. 4 we present the results in the 


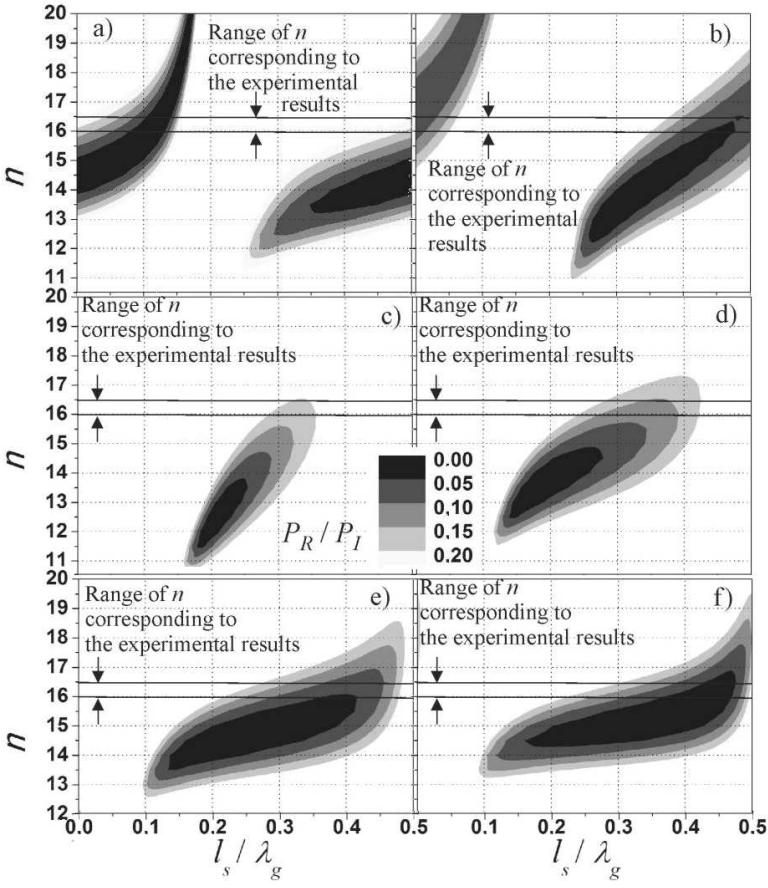

Fig. 3. Calculated tuning characteristics of MPM for different values of height of the reduced section waveguide $b_{1}$. (a) $b_{1}=b, w_{1}=a$, without iris, (b) $b_{1}=0.9 b$, $w_{1}=a$, without iris, (c) $b_{1}=0.6 b, w_{1}=a$, without iris, (d) $b_{1}=0.4 b, w_{1}=a$, without iris, (e) $b_{1}=0.3 b$, $w_{1}=a$, without iris, (f) $b_{1}=0.25 b, w_{1}=a$, without iris.

range of 0 to 0.25 in order to focus on most wanted values of $P_{R} / P_{I}$. The tuning characteristics of MPM were calculated for several values of height of reduced section $b_{1}(b, 0.9 b, 0.8 b, 0.7 b, 0.6 b, 0.5 b, 0.4 b, 0.3 b, 0.25 b)$, several values of ridge width $w_{1}\left(w_{1}=a, w_{1}=0.8 a, w_{1}=0.6 a\right)$, and several values of iris width $d(d=a, d=0.7 a$, $d=0.625 a$ ). In this paper we present the tuning characteristics for selected cases of construction of MPM. We assumed those plasma parameters for which results of the numerical analysis were similar to the experiment at the beginning of optimization. The results of the numerical analysis show that $P_{R} / P_{I}$ is below 0.05 in wide range of movable plunger position $l_{s}$ for $b_{1}=0.3 b, w_{1}=0.8 a$, and with iris width $d=0.625 a$ (Fig. 4c).

\section{Conclusions}

The tuning characteristics of the presented MPM depend not only on plasma parameters but also on dimensions of MPM. We examined how the height of the reduced-height section $b_{1}$, width of the ridge $w_{1}$, and width of the iris $d$ influence on the tuning characteristics of the investigated MPM.

The numerical investigation presented in this paper shows that application of a ridge waveguide with a width $w_{1}=0.8 a$, height and inductive iris of a width $d=0.625 a$ can improve power efficiency of MPM because the value $P_{R} / P_{I}$ can be lowered below 0.05 in the wide range of the movable plunger positions.

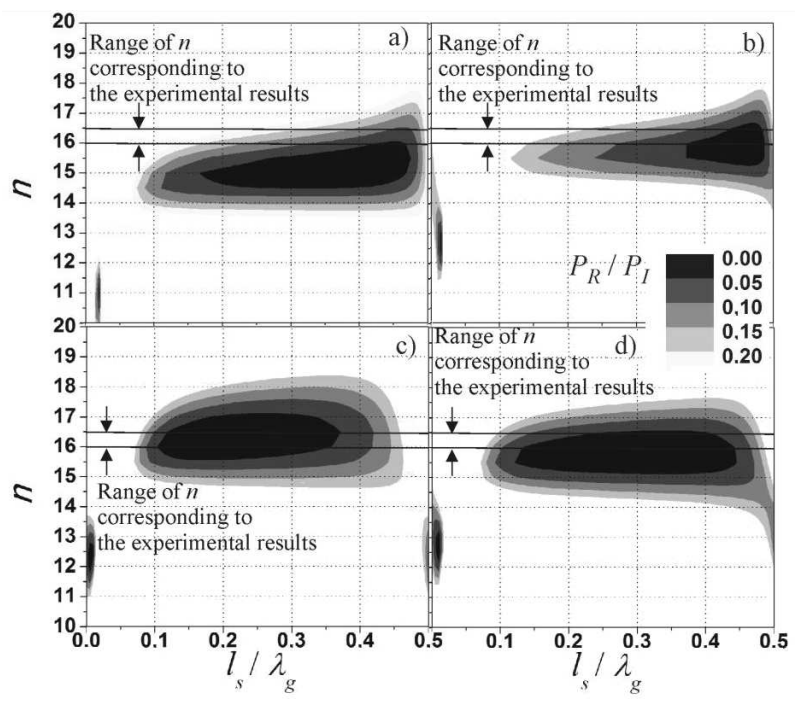

Fig. 4. Calculated tuning characteristics of MPM for $b_{1}=0.3 b$ and different values of width of the ridge $w_{1}$ and width of iris $d$. (a) $w_{1}=0.6 a$, without iris, (b) $w_{1}=$ $0.8 a$, without iris, (c) $w_{1}=0.8 a$, with iris, $d=0.625 a$, (d) $w_{1}=0.8 a$, with iris, $d=0.7 a$.

\section{Acknowledgments}

This research was supported by The National Centre for Research and Development (NCBiR) under programme NR14-0091-10/2010.

\section{References}

[1] M. Jasiński, M. Dors, J. Mizeraczyk, Plasma Chem. Plasma Proc. 29, 363 (2009).

[2] M. Jasiński, J. Mizeraczyk, Z. Zakrzewski, J. High Temp. Mater. Proc. 6, 317 (2002).

[3] M. Dors, T. Izdebski, B. Hrycak, J. Mizeraczyk, M. Hołub, K. Bonisławski, Przeglad Elektrotechniczny 07a, 155 (2012).

[4] Z. Kolacinski, L. Szymanski, G. Raniszewski, S. Wiak, Przeglad Elektrotechniczny 10b, 149 (2012).

[5] M. Jasiński, M. Dors, H. Nowakowska, J. Mizeraczyk, Chemicke Listy 102, 1332 (2008).

[6] M. Jasiński, M. Dors, J. Mizeraczyk, Europ. Phys. J. D 54, 179 (2009).

[7] J. Helszajn, Ridge Waveguides and Passive Microwave Components, Institution of Engineering and Technology, London U.K. 2000.

[8] Z. Zakrzewski, D. Czylkowski, M. Jasiński, M. Moisan, D. Guerin, C. Larquet, J.C. Rostaing, Microwave Plasma Exciters, US Patent, US7799119 (2010).

[9] H. Nowakowska, M. Jasiński, J. Mizeraczyk, in: Proc. Comsol Conf., Hannover, Ed.: A. Jungr, Comsol AB, Hannover 2008.

[10] M. Sobański, M. Jasiński, J. Mizeraczyk, Przeglad Elektrotechniczny 10b, 135 (2012) (in Polish).

[11] R.E. Collin, Field Theory of Guided Waves, McGrawHill, New York 1960.

[12] H. Nowakowska, M. Jasiński, J. Mizeraczyk, Przeglad Elektrotechniczny 07, 84 (2010) (in Polish). 